\title{
Mécanique spontanée du son
}

\section{Maurines}

\section{(2) OpenEdition}

Journals

Édition électronique

URL : http://journals.openedition.org/trema/2342

DOI : 10.4000/trema.2342

ISSN : 2107-0997

\section{Éditeur}

Faculté d'Éducation de l'université de Montpellier

\section{Édition imprimée}

Date de publication : 1 mai 1993

Pagination : 77-91

ISSN : 1167-315X

\section{Référence électronique}

L. Maurines, « Mécanique spontanée du son », Tréma [En ligne], 3-4 | 1993, mis en ligne le 01 mai 1993, consulté le 20 avril 2019. URL : http://journals.openedition.org/trema/2342 ; DOI : 10.4000/ trema.2342

Ce document a été généré automatiquement le 20 avril 2019

Trema 


\title{
Mécanique spontanée du son
}

\author{
L. Maurines
}

1 Le travail présenté ici fait partie d'une recherche en cours portant sur les raisonnements spontanés en acoustique. Il vise à analyser les tendances d'ensemble des raisonnements d'élèves et d'étudiants susceptibles de se manifester dans des populations diverses afin d'en proposer une description organisée et prédictive, à alimenter une réflexion sur les objectifs pédagogiques à poursuivre et à permettre la construction d'outils d'enseignement adaptés au traitement des difficultés observées.

2 Il fait suite à une recherche analysant les difficultés rencontrées par les élèves lors de l'étude de la propagation d'un signal transversal sur une corde (Maurines 1986, 1992). Il cherche à savoir si les raisonnements spontanés de type mécaniste qui sont à l'origine de certaines de ces difficultés se manifestent aussi pour des signaux non visibles, se propageant avec une vitesse plus élevée. Avant de montrer que dans les situations étudiées c'est le cas, nous présenterons en quelques mots la problématique générale de ces recherches sur les signaux puis récapitulerons brièvement les résultats obtenus pour la propagation d'un signal sur une corde et l'interprétation qui en est donnée.

\section{Problématique, méthodologie}

3 Les thèmes spécifiques de ces recherches sont en partie inspirés des programmes de la classe de première $\mathrm{S}$, classe où est abordée pour la première fois l'étude des ondes ( $\mathrm{du}$ moins jusqu'à cette année puisqu'à la rentrée 93 le son sera étudié en classe de seconde). La partie «ondes » du programme de première $S$ débute par la propagation d'un signal. L'approche utilisée à ce niveau est essentiellement expérimentale, descriptive et macroscopique. Il s'agit en particulier de présenter aux élèves de nombreuses expériences de propagation de signaux variés (sur une corde, un ressort, de l'eau, signaux sonores et lumineux), de les étudier à l'aide des graphes des deux descriptions possibles d'un phénomène de propagation (cf encadré $1, \mathrm{p}$. 86), à savoir :

- la description spatiale qui donne l'état du milieu en chaque point de l'espace à un instant donné, 
- la description temporelle qui donne l'évolution de l'état du milieu au cours du temps en un point donné de l'espace.

4 Cette étude graphique est faite principalement pour la propagation d'un ébranlement sur une corde en supposant le milieu " parfait » (un signal à une dimension se propage alors sans déformation).

A ce niveau d'enseignement, on peut attendre des élèves :

- de comprendre le rôle de la source, du milieu, des frottements,

- de savoir de quoi dépendent les grandeurs physiques caractérisant le phénomène (en particulier, la vitesse de propagation $\mathrm{V}$, la durée du signal $\mathrm{T}$, la largeur du signal L, l'amplitude du signal $\mathrm{H}$ ),

- de savoir utiliser des relations mettant en jeu simultanément trois grandeurs physiques(en particulier, la relation L=VT reliant la largeur du signal à la vitesse de propagation et à la durée du signal),

- de dessiner correctement les graphes de la description spatio-temporelle. Au niveau universitaire, on peut attendre en plus des étudiants de maîtriser le formalisme mathématique de l'équation des ondes et de ses solutions.

6 Après une enquête préliminaire (quelques interviews et questionnaires), la recherche sur le signal sur la corde a été focalisée sur trois problématiques différentes (pour les élèves, de quoi dépendent les trois grandeurs V, L, T ? Comment se comportent-ils dans des situations à plusieurs variables? Quelles difficultés rencontrent-ils avec la description spatio-temporelle ?). Elles ont été explorées à l'aide d'une vingtaine de questionnaires papier-crayon.

7 L'étude sur le son présentée ici a été conduite en élaborant des questionnaires (une dizaine environ) se rapprochant de ceux conçus pour la corde et apportant des éléments de réponse à la première problématique.

Dans cette communication, seules les grandeurs V et T seront abordées, aussi bien pour la corde que pour le son. La population interrogée sur la corde est de l'ordre de 700 élèves avant enseignement sur les ondes (classes de seconde, première scientifique et technique, terminale technique) et de 600 après (classes de première et terminale scientifique, trois premières années d'université scientifique). Celle interrogée sur le son est de l'ordre de 550 élèves avant enseignement sur les ondes (classes de troisième, seconde, première scientifique) et de 150 après (classes de terminale scientifique, deux premières années d'université scientifique).

9 Les raisonnements dégagés ne sont que des tendances d'ensemble et non ceux d'un élève particulier. Ils se manifestent à des degrés divers après enseignement. L'énoncé des questionnaires est schématisé. Les pourcentages des différentes réponses sont calculés sur le nombre d'élèves interrogés, accompagnés de E0, El, selon le type de population interrogée (E0 avant enseignement, E1 après enseignement).

\section{Propagation d'un signal sur une corde}

Nous allons montrer que :

- les élèves expliquent la propagation d'un signal sur une corde comme si la forme visible qui se déplace, « la bosse », était un objet matériel créé et mis en mouvement par la source (la main qui tient l'extrémité de la corde),

- leur raisonnement s'appuie sur un concept hybride appelé « capital ». 


\section{La corde emmagasine un « capital » que la source lui communique et met en mouvement}

11 Intéressons-nous aux résultats d'une question (cf. encadré 2) demandant s'il y a un moyen de bouger la main pour qu'un point de la corde bouge plus tôt.

Alors que la vitesse de propagation ne dépend que du milieu, $60 \%$ des élèves interrogés avant enseignement $(\mathrm{N}=42)$ et $75 \%$ après $(\mathrm{N}=16)$ répondent que la vitesse de propagation dépend de ce que fait la main. Les nombreuses justifications dynamiques indiquent que c'est la force exercée par la main pour créer le signal qui importe. Plus celle-ci est grande, plus la vitesse l'est :

« la bosse se déplacera de plus en plus vite', si le geste est vif ».

" la vitesse dépend de la force avec laquelle il a bougé la main ».

13 La cause (la secousse donnée par l'expérimentateur) et l'effet (la bosse et son déplacement) sont amalgamés: en effet, tout semble se passer comme si cette force restait attachée au signal et se déplaçait avec lui :

«si l'intensité de la force propagée est plus forte alors la bosse aussi se répandra plus vite».

Certains schémas fournis en plus des explications renforcent cette hypothèse : la force qui y est représentée, est localisée sur la bosse (cf. encadré 2).

La source semble donc communiquer quelque chose à la corde lors de la formation du signal, quelque chose qui se déplace et reste localisée dans la bosse. Les élèves l'appellent le plus souvent "force», mais c'est en réalité une notion hybride, mélange de force, d'énergie, de vitesse. Cette force qui n'a rien à voir avec une force au sens de la physique a été appelée CAPITAL, car, tout comme un capital, la corde l'emmagasine.

\section{Le capital a une forme et la bosse le matérialise}

La source met non seulement en mouvement le capital mais le fabrique comme si c'était un objet matériel. Elle lui donne une forme que matérialise la bosse. C'est ce qui apparaitt sur les résultats obtenus à un questionnaire (cf. encadré 3) demandant de comparer la vitesse de trois signaux de formes différentes. Alors que les vitesses de propagation sont identiques, $87 \%$ des élèves interrogés avant enseignement $(\mathrm{N}=93)$ et $41 \%$ après $(\mathrm{N}=27)$ répondent qu'elles sont différentes. Les nombreuses justifications dynamiques faisant intervenir la force exercée par la main et communiquée au signal, c'est à dire le CAPITAL, montrent que la «bosse » le matérialise, que la forme du signal (plus précisément son amplitude) et la vitesse de propagation varient dans le même sens que lui :

« la C va le plus vite. Car la force fournie par le bras de l'enfant modifie la forme de la bosse et la vitesse. Donc plus la force est intense, plus la forme de la bosse sera grande et plus la vitesse sera grande »,

«[...] cela dépend de la force avec laquelle le mouvement a été fait. Cela se voit selon la grandeur de la bosse. Elle est le reflet de cette force engagée par l'enfant pour arriver à ce résultat $[\ldots]$ » 


\section{Le capital peut varier au cours du temps}

17 Le mouvement de la bosse et son existence sont perçus par les élèves comme les deux facettes d'un seul objet, le capital, Lorsque celui-ci varie, par suite de frottements par exemple, les deux grandeurs physiques associées à ces deux facettes $(\mathrm{V}$ et $\mathrm{H})$ varient simultanément et dans le même sens que lui. Ainsi à une question demandant si la vitesse de propagation d'une bosse qui disparaît avant d'atteindre l'autre extrémité de la corde est constante au cours du temps, $68 \%$ des élèves interrogés avant enseignement $(\mathrm{N}=56)$ et $55 \%$ après $(\mathrm{N}=42)$ répondent qu'elle diminue car pour eux une diminution d'amplitude indique une diminution du capital et donc de vitesse de propagation :

« la hauteur faiblit car l'action de la main s'atténue»,

«si la bosse disparaît, c'est que la force qui la «faisait» disparaît. Pendant ce temps, la vitesse diminue. »

\section{Conséquences d'un raisonnement en terme de capital}

Nous venons de voir que le raisonnement spontané s'appuie sur la notion de capital, c'està-dire d'un objet créé et mis en mouvement par la source, matérialisé par la bosse. Ce concept hybride conduit les élèves et étudiants en particulier :

- à appliquer à la bosse qui se déplace une « mécanique » de l'objet matériel (la vitesse de propagation du signal dépend de la source et peut varier au cours du temps). Cette mécanique spontanée du signal présente des ressemblances avec la mécanique spontanée du solide analysée par L. Viennot et E. Saltiel. Tout comme le capital de force introduit par L. Viennot, le capital est mis en mouvement par la source, reste localisé dans la bosse, peut diminuer au cours du temps et enfin détermine la vitesse de propagation.

- à une interdépendance entre le mouvement transversal d'un point du milieu et la vitesse de propagation, et donc à des liens entre les trois grandeurs physiques H, V, T. Ainsi nous venons de voir que les élèves peuvent déduire la vitesse de propagation du mouvement de la source et de l'amplitude du signal. Dans d'autres situations, ils relient la durée du signal à la vitesse de propagation ou à l'amplitude du signal : c'est ainsi que la durée d'un signal n'est pas conservée lors d'une transmission d'un milieu à un autre.

\section{Propagation d'un signal sonore}

19 Nous allons voir à présent que les résultats obtenus pour le son peuvent donner lieu à la même interprétation.

\section{Vitesse du son et source}

La première question du questionnaire de l'encadré 4 porte sur la comparaison des vitesses de deux signaux sonores émis par des sources d'amplitudes différentes.

Pour $40 \%$ des élèves interrogés ( $\mathrm{E} 0, \mathrm{~N}=62)$, l'auditeur ne commence pas à entendre les sons émis par les deux sources au même instant. Pour la majorité d'entre eux (39\% sur la population totale), la source de plus grande amplitude sonore est entendue en premier. 
Les justifications explicitent le lien entre l'amplitude sonore et la vitesse de propagation (92\% des réponses justifiées) :

" elle entend Pierre en premier car il chante plus fort ",

" elle entend Pierre en premier car le son étant plus élevé est projeté plus vite »

"c'est Pierre qu'elle va entendre en premier car comme il chante plus fort le son arrive plus vite à son pavillon ».

Ces deux dernières justifications montrent bien que comme la main donne une vitesse d'autant plus grande à la bosse que celle-ci est haute, la voix donne une vitesse d'autant plus grande au son que celui-ci est fort.

\section{Vitesse du son et amplitude du signal}

La liaison entre l'amplitude du signal et la vitesse de propagation apparaît de nouveau sur les résultats obtenus aux deux premières questions du questionnaire de l'encadré 5 . Celui-ci porte sur la comparaison de deux sons émis par des sources identiques, l'un se propageant à l'air libre, l'autre étant guidé par un tube.

24 A la première question portant sur l'intensité sonore, $89 \%$ des élèves interrogés (E0, $\mathrm{N}=28$ ) répondent que le son guidé est plus fort. $92 \%$ des réponses sont justifiées et invoquent le fait que le son est " coincé » ( $72 \%$ d'apparition), qu'il n'y a pas de perte $(20 \%)$, que dans le tube le son n'est pas gêné comme à l'air libre par des obstacles, une résistance etc ... (28\%).

A la deuxième question portant sur la vitesse de propagation, $79 \%$ des élèves ( $\mathrm{E} 0, \mathrm{~N}=28$ ) répondent que le son guidé par le tube n'est pas entendu au bout du même intervalle de temps que le son se propageant à l'air libre. $68 \%$ d'entre eux (soit $54 \%$ sur la population totale) pensent que la vitesse du son guidé est plus grande. Les justifications sont de même nature que celles données pour l'intensité :

" Jean reçoit plus de « son ». II l'entend plus tôt car le son lui parvient un peu plus tôt grâce au tube qui accompagne directement les ondes évitant ainsi les pertes »,

«l'intensité du son n'est pas la même, elle est plus grande, car dans cette situation rien ne perturbe la propagation du son, puisque le son est isolé. Jean commence à entendre Pierre plus tôt puisque rien ne freine ou perturbe le passage du son de Pierre à Jean ».

L'effectif de la population interrogée ne permet pas de faire une analyse plus poussée de la nature des justifications et de leur répartition. Quoi qu'il en soit, une corrélation entre les réponses obtenues aux deux questions fait apparaître un lien privilégié du type : «V augmente avec $\mathrm{H} »$ ( $43 \%$ de réponses).

\section{Vitesse du son et propagation}

Les résultats précédents rappellent ceux obtenus pour la corde : la vitesse de propagation dépend de la source et de l'amplitude du signal. Pour rendre compte de ces liens, le même type de concept hybride (le capital) peut être introduit comme support du raisonnement des élèves. Mélange d'énergie, d'intensité, de vitesse, il est communiqué par la source au milieu et est substantialisé par le " corpuscule sonore ». Il peut se modifier au cours de la propagation (par exemple si le milieu est à trois dimensions) entraînant la variation simultanée de l'amplitude et de la vitesse du son. Le questionnaire de l'encadré 6 portant 
sur ce qui est entendu lors de la propagation d'un signal en deux points situés à des distances différentes de la source le met en évidence. celui de support passif dans lequel se déplace une perturbation dont les caractéristiques sont fixées par la source. Nous allons montrer qu'à la limite, dans le cas du son, ce milieu n'est pas utile à la propagation mais qu'il est plutôt une gêne.

\section{Capital et interdépendance entre $V$ et ce qui est entendu en un point de l'espace}

Conditionnant à la fois l'existence et le déplacement du son, le capital conduit à une interdépendance entre la vitesse de propagation et ce qui est entendu en un point de l'espace, c'est-à-dire à des liens entre les trois grandeurs V, H, T. Ainsi nous venons de voir que les élèves peuvent déduire la vitesse du son de l'amplitude sonore. Dans d'autres situations, ils relient la durée sonore à l'amplitude sonore ou à la vitesse de propagation. Le lien entre $\mathrm{H}$ et $\mathrm{T}$ est illustré ici grâce aux trois questionnaires précédemment présentés. L'encadré 7 récapitulant les résultats obtenus montre que pour un nombre important d'élèves, la durée sonore dépend de l'amplitude sonore, le lien majoritaire étant du type : « $\mathrm{T}$ augmente avec $\mathrm{H}$ ».

Une analyse poussée de la nature des justifications et de leur répartition ne peut être faite. Plusieurs questions méritent d'être approfondies, entre autres : le lien entre $\mathrm{H}$ et $\mathrm{T}$ n'indiquerait-il pas que les élèves répondent sur la durée du mouvement de la source vibrante et non pas sur la durée d'un signal sonore donné ? le lien entre $\mathrm{H}$ et $\mathrm{T}$ ne serait-il pas induit par la perception de l'oreille?

Il est à noter que la non dépendance de la vitesse de propagation avec ce qui est entendu en un point de l'espace est une question que nos prédécesseurs ont explorée. A la fin du dix-huitième siècle, Gassendi et Nollet ont expérimenté sur le son et ont montré respectivement que sa vitesse ne dépendait pas de son amplitude et de sa fréquence.

\section{Rôle du milieu}

Tout d'abord la question se pose de savoir si un son peut se propager dans le vide. Le questionnaire de l'encadré 8 explore ce point.

Bien qu'un nombre important d'élèves, $40 \%(E 0, N=62)$, pensent qu'un astronaute en orbite ne peut entendre la catastrophe se produisant sur la lune, seuls $32 \%$ d'entre eux (soit $13 \%$ sur la population totale) le justifient par le fait qu'en l'absence d'air le son ne peut se propager : 
«non, car sans «air» les bruits ne viennent pas à nos oreilles et sur la lune, il n'y a pas assez, voire pas d'air du tout»,

« l'astronaute ne pourrait pas l'entendre car il n'y a pas d'air et seul l'air conduit le son». invoquent la distance entre lui et la lune: celle-ci est suffisamment petite pour que l'astronaute entende. Les résultats de la deuxième question confirment que le critère retenu par ces élèves est la distance : le nombre d'élèves pensant que la catastrophe ne peut être entendue de la terre augmente ainsi que les justifications liées à la distance, à présent devenue trop grande :

« nous ne l'entendrons pas sur terre car la distance entre la lune et la terre est trop grande. L'astronaute l'entendra peut-être, mais à quelle distance est-il en orbite? »

La question se pose maintenant de savoir si pour les élèves un son peut se propager dans un milieu matériel autre que l'air. Le questionnaire de l'encadré 9 répond à cette question et permet de confirmer les résultats obtenus à propos de la propagation dans le vide. Il demande de comparer la propagation d'un son émis par des sources identiques dans des « milieux » différents : de l'air (à des pressions et des températures différentes), un autre gaz (hydrogène), un liquide (eau), un solide (acier) et le vide. On retrouve sur les résultats regroupés dans le premier tableau que beaucoup d'élèves, $74 \%$ (E0, N=39) pensent que le son peut se propager dans le vide. La propagation est par contre impossible pour un nombre significatif des élèves dans un liquide et dans un solide. Les résultats ainsi que les justifications données semblent indiquer que plus le milieu est dense, plus il gêne la propagation.

\footnotetext{
« oui pour le vide : le son ne rencontre aucune opposition ».

" oui, sauf l'acier plein parce que le son ne pourra pas traverser le tube plein pour être enregistré, et l'eau»,

« oui pour l'air, le vide, l'hydrogène car il n'y a pas la présence d'isolant comme l'eau dans le tube $5 »$,

« sauf acier, car c'est un métal compact qui ne laisse passer ni l'air ni l'eau donc les sons ne pourront passer».
}

38 Les réponses obtenues à la question sur les vitesses de propagation confirment que la propagation est d'autant plus difficile que le milieu est dense. Bien que $82 \%$ des élèves pensent que la vitesse de propagation du son dépend du milieu, le classement donné ne correspond pas à la réalité (deuxième tableau) : un son se propage plus vite dans le vide que dans l'eau ou l'acier:

« non, certaines matières ralentissent. Classement donné par ordre de vitesse décroissant : 3,1,7,4,2,5,6 ( air, vide, hydrogène, air, eau, acier) ».

La phrase : « tube où on a fait le vide " pouvant ne pas avoir été comprise par les élèves et induire un nombre élevé de réponses du type "le son se propage dans le vide», une deuxième passation de la première question de ce questionnaire a eu lieu. Dans cette version, la mention «il n'y a rien " complète cette phrase et seuls quatre tubes sont présentés (air, eau, acier, vide). Le nombre d'élèves pensant que le son peut se propager dans le vide a diminué tout en restant significatif ( $31 \%, \mathrm{E} 0, \mathrm{~N}=26)$. Bien que le nombre de réponses du type « le son se propage dans l'eau » soit plus élevé, il ne signifie pas que les élèves conçoivent le milieu comme support de la propagation d'une énergie: des justifications semblent indiquer qu'il peut se déplacer dans son ensemble ou que la propagation est possible car le milieu contient un gaz (air ou oxygène) : 
« air : oui, c'est comme un souffle

eau : oui, car lorsque l'eau bouge ça s'entend

acier : non, parce que l'acier ne fait pas de bruit, reste immobile

vide : non, il n'y a rien »,

« air : oui, l'air conduit le son jusqu'au microphone

eau : oui, il y a de l'oxygène dans l'eau

vide : non, il n'y a pas d'air pour conduire le son

acier : oui, l'air conduit le son ».

\section{Conclusion}

Bien que la recherche sur les raisonnements spontanés en acoustique ne soit pas terminée et que l'étude présentée ici demande à être affinée, un premier bilan permet de dire qu'il existe des similitudes entre les réponses obtenues pour la propagation d'un signal sur une corde et celles obtenues pour la propagation d'un signal sonore. Nous rencontrons pour les deux phénomènes des tendances vers un raisonnement mononotionnel en terme d'objet. Ce type de raisonnement ne semble donc pas provenir des caractéristiques visuelles du signal sur la corde (localisé, vitesse de propagation faible si bien qu'il peut être suivi des yeux) mais trouve sans doute son origine clans l'expérience commune. D'autres travaux dans d'autres domaines de la physique (mécanique (L. Viennot, E. Saltiel et J-L. Malgrange), électricité (J-L. Closset), thermodynamique (S. Rozier et L. Viennot)) vont également dans ce sens.

41 La recherche sur la corde a conduit à des propositions pédagogiques. Nombre d'entre elles pourraient être reconduites pour le son. Soulignons l'intérêt d'utiliser en classe les questionnaires construits pour ces recherches et de mener une comparaison par ressemblances et différences entre le mouvement d'un solide et la propagation d'un signal.

\section{Encadré $n^{\circ} 1$}

Description spatiale

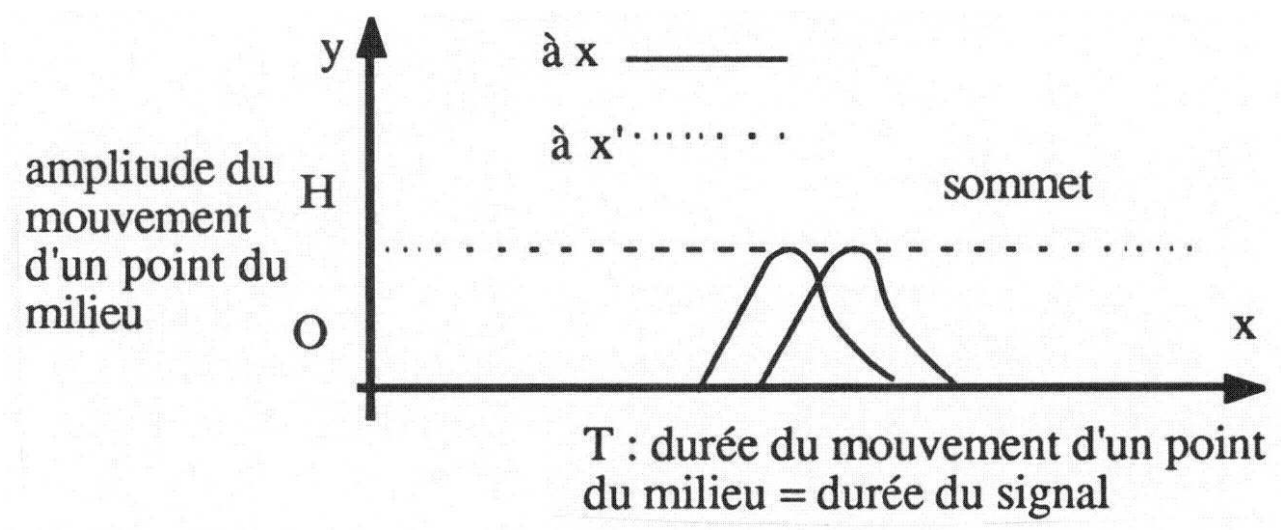


Description temporelle

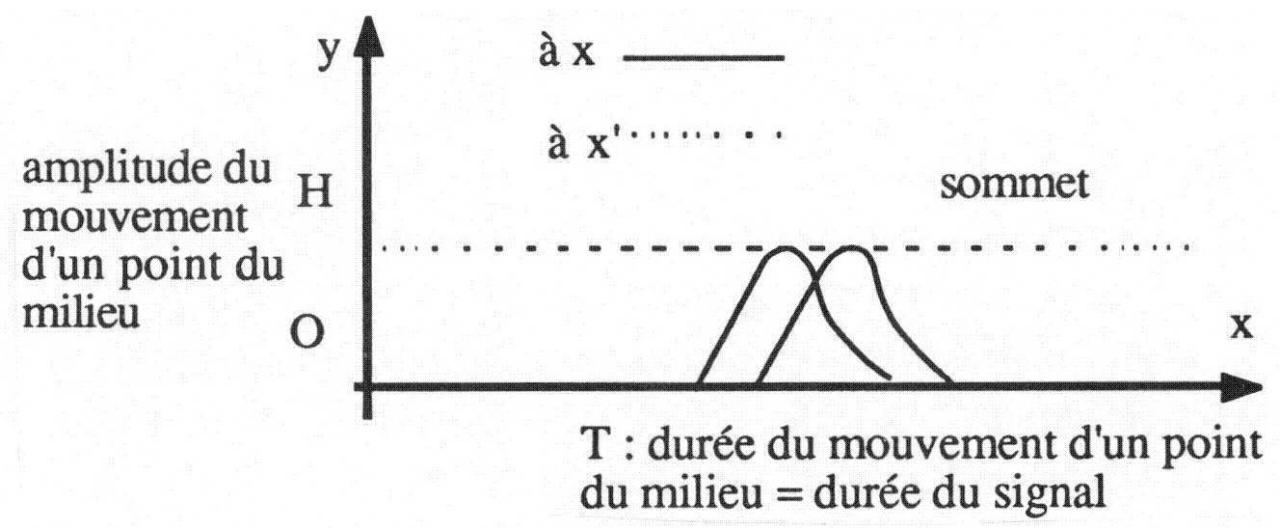

\section{Encadré $\mathrm{n}^{\circ} 2$}

Enoncé simplifié du questionnaire : en bougeant la main, on observe la forme suivante à l'instant $t$ :

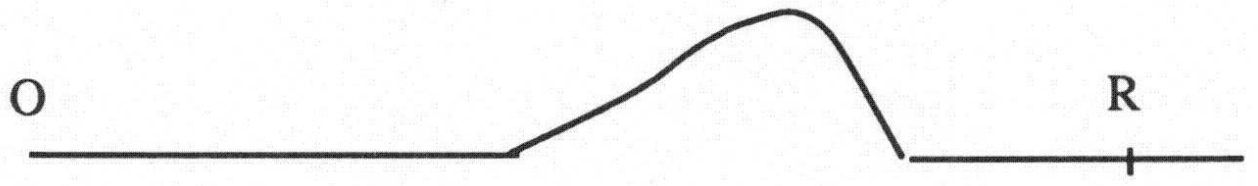

Y a-t-il une façon de bouger la main pour que la forme atteigne R plus tôt ?

Oui, laquelle? Non, pourquoi?

42 Exemples de réponses:

"plus le geste est vif, plus la bosse ira vite », "... on peut dire aussi qu'il se forme des vagues qui bougent à cause de la force $\mathrm{F}$

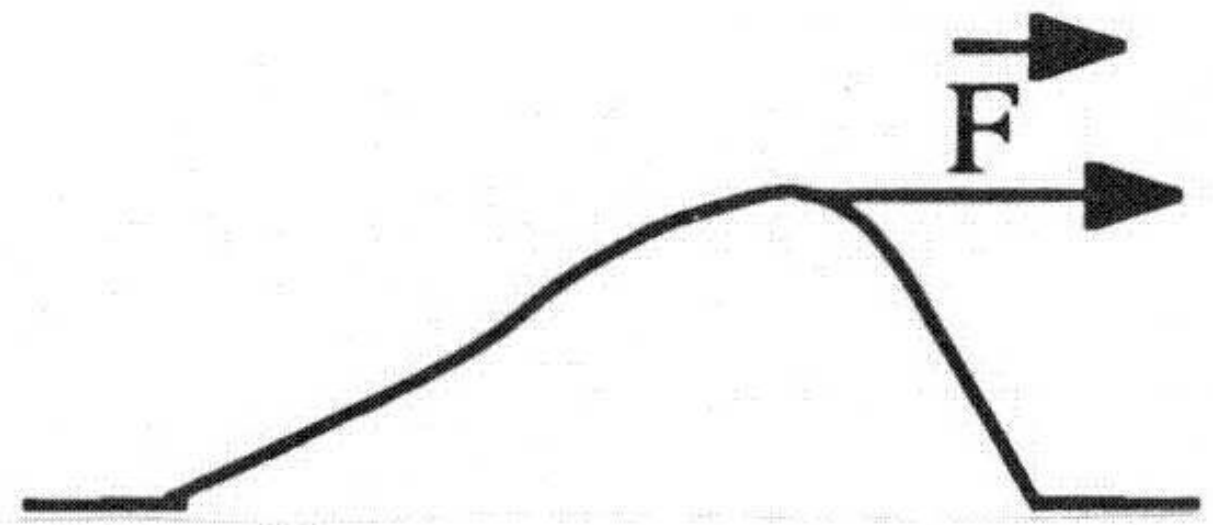

\section{Encadré $\mathrm{n}^{\circ} 3$}


Enoncé : ces trois signaux ont-ils la même vitesse? Oui, pourquoi ? Non, pourquoi ? Quel est le plus rapide, le plus lent?

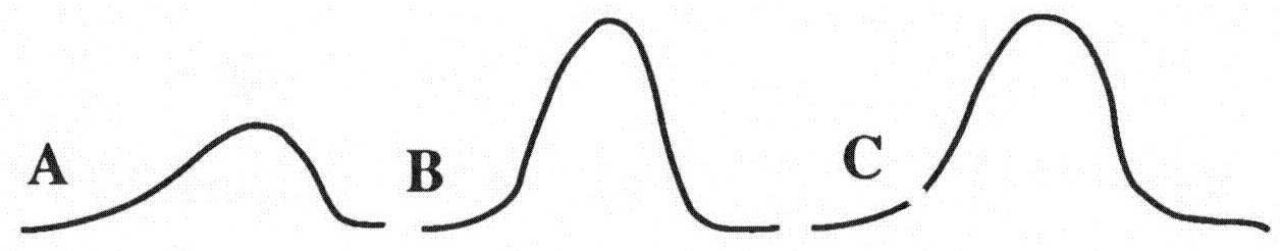

\section{Encadré $n^{\circ} 4$}

Enoncé : trois enfants sur une route rectiligne.

P chante M c M chante

P et J chantent un "la" pendant 1 seconde mais P chante plus fort que J.

question 1 : est-ce que $\mathrm{M}$ commence à entendre $\mathrm{P}$ et $\mathrm{J}$ au même instant ? Oui, pourquoi ? Non, qui entend $M$ en premier ? Pourquoi ?

question 2 : est-ce que $M$ entend $P$ et $J$ pendant la même durée? Oui, pourquoi ? Non, qui entend $\mathrm{M}$ le plus longtemps ? Pourquoi?

Résultats :

\begin{tabular}{|l|c|c|c|}
\hline & V différentes & Videntiques & rien \\
\hline E0, $\mathrm{N}=62$ & $\mathbf{4 0} \%$ & $42 \%$ & $18 \%$ \\
troisième & V augmente avec H: & & \\
& $\mathbf{3 9} \%$ & & \\
\hline
\end{tabular}

\section{Encadré $n^{\circ} 5$}

Enoncé : comparez ce qu'entend $J$ dans les deux situations suivantes, où $P$ parle de la même façon.

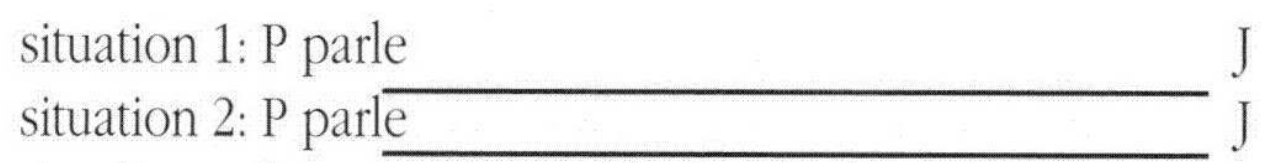

P parle par l'intermédiaire d'un long tube en acier.

Précisez en particulier :

1. si dans la situation 2, l'intensité sonore est plus grande, plus petite, identique à l'intensité sonore dans la situation 1 ? Pourquoi?

2. si dans la situation $2, \mathrm{~J}$ commence à entendre $\mathrm{P}$ au bout du même intervalle de temps que dans la situation 1, ou si c'est plus tôt ou plus tard ? Pourquoi?

3. si dans la situation 2 , J entend $P$ pendant la même durée que dans la situation 1 , ou si c'est pendant moins longtemps ou plus longtemps? Pourquoi ?

Résultats à la question 1 sur l'intensité sonore :

\begin{tabular}{|l|c|c|}
\hline & intensités différentes & intensités identiques \\
\hline $\begin{array}{l}\mathrm{E} 0, \mathrm{~N}=28 \\
\text { première S }\end{array}$ & $89 \%$ & $11 \%$ \\
\hline
\end{tabular}


Résultats à la question 2 sur les vitesses de propagation:

\begin{tabular}{|l|c|c|}
\hline & vitesses différentes & vitesses identiques \\
\hline $\mathrm{E} 0, \mathrm{~N}=28$ & $\mathbf{7 9} \%$ & $11 \%$ \\
première $\mathrm{S}$ & $\mathrm{V} 2>\mathrm{V} 1: \mathbf{5 4} \%$ & \\
& $\mathrm{~V} 2<\mathrm{V} 1: 25 \%$ & \\
\hline
\end{tabular}

\section{Encadré $n^{\circ} 6$}

Enonce

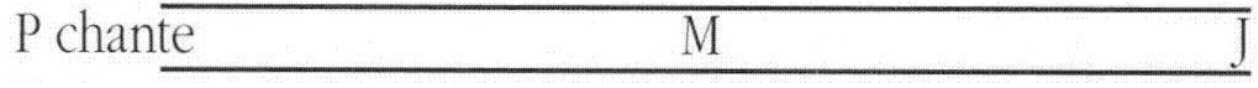

$P$ chante un «la » pendant une seconde.

question 1 : Est- ce que $M$ et $\mathrm{J}$ commencent à entendre le son au même instant? Oui, pourquoi ? Non, pourquoi?

Si vous avez répondu non à la question précédente répondez aux questions suivantes :

question 2 : Le son met-il autant de temps pour aller de $\mathrm{P}$ à $\mathrm{M}$ que pour aller de $\mathrm{M}$ à $\mathrm{J}$ ? Oui, pourquoi ? Non, pourquoi ?

question 3 : Est- ce que $\mathrm{M}$ et $\mathrm{J}$ entendent $\mathrm{P}$ pendant la même durée ? Oui, pourquoi ? Non, pourquoi ? Quel est celui qui entend P pendant la plus grande durée ? Pourquoi ?

Résultats :

résultats à la question 1 sur les instants du début d'audition du son :

\begin{tabular}{|l|c|c|c|}
\hline & différents & identiques & rien \\
\hline $\begin{array}{l}\mathrm{E} 0, \mathrm{~N}=25 \\
\text { troisième }\end{array}$ & $80 \%$ & $12 \%$ & $8 \%$ \\
\hline
\end{tabular}

résultats à la question 2 sur les durées de propagation sur les deux parcours de même longueur:

\begin{tabular}{|l|c|c|c|}
\hline & identiques & différentes & rien \\
\hline $\begin{array}{l}\mathrm{E} 0, \mathrm{~N}=25 \\
\text { troisième }\end{array}$ & $48 \%$ & $\mathbf{3 2} \%$ & $20 \%$ \\
\hline
\end{tabular}

\section{Encadré $\mathrm{n}^{\circ} 7$}

- Résultats sur les durées d'audition ; résultats à la question 2 de l'encadré 4 :

P chante M c Mante

\begin{tabular}{|c|c|c|c|}
\hline & durées différentes & durées identiques & rien \\
\hline $\begin{array}{c}\mathrm{E} 0, \mathrm{~N}=62 \\
\text { troisième }\end{array}$ & $\begin{array}{c}\mathbf{4 5 \%} \\
\text { T augmente } \\
\text { avec } \mathrm{H} \mathbf{3 2} \%\end{array}$ & $34 \%$ & $21 \%$ \\
& & & \\
\hline
\end{tabular}


«P car la voix forte reste assez longtemps »

«P car il chante plus fort. II y a une résonance qui fait durer plus longtemps la voix de $P$ »

- Résultats à la question 3 de l'encadré 5 :

P parle

P parle

\begin{tabular}{|l|c|c|c|}
\hline & durées différentes & durées identiques & rien \\
\hline $\begin{array}{l}\text { E0 }, \mathrm{N}=28 \\
\text { première S }\end{array}$ & $\begin{array}{c}\mathbf{5 4 \%} \\
\text { Taugmente } \\
\text { avec } \mathrm{H} \mathbf{3 6 \%}\end{array}$ & $21 \%$ & $25 \%$ \\
& & & \\
\hline
\end{tabular}

«P car le son est soumis à des vibrations et est intensifié »

- Résultats à la question 3 de l'encadré 6 :

P chante M

\begin{tabular}{|l|c|c|c|}
\hline & durées différentes & durées identiques & rien \\
\hline $\begin{array}{l}\text { E0 }, \mathrm{N}=25 \\
\text { troisième }\end{array}$ & $\begin{array}{c}\mathbf{6 8} \% \\
\mathrm{~T} \text { diminue } \\
\text { avec } \mathrm{H} \mathbf{4 0 \%}\end{array}$ & $24 \%$ & $8 \%$ \\
& & \\
\hline
\end{tabular}

«M entend pendant plus longtemps car le son s'atténue et devient plus court »

«M entend pendant la plus grande durée car c'est la deuxième et que le son est tout frais »

«car si le son ralentit quand $\mathrm{J}$ le recevra, il n'entendra pas tout »

\section{Encadré $\mathrm{n}^{\circ} 8$}

Enoncé: si une catastrophe se produisait sur la lune (par exemple, un séisme ou tremblement de «lune») un astronaute en orbite autour de la lune l'entendrait-il? Pourquoi ? Pourrions-nous l'entendre sur terre ? Pourquoi ?

Résultats à la question sur l'astronaute, $A$ :

\begin{tabular}{|l|c|c|}
\hline & A n'entend pas & A entend \\
\hline $\begin{array}{l}\mathrm{E} 0, \mathrm{~N}=62 \\
\text { troisième }\end{array}$ & $40 \%$ & $\mathbf{3 5 \%}$ \\
\hline
\end{tabular}

$13 \%$ sur l'ensemble note que A n'entend pas car sans air, il n'y a pas de son Résultats à la question sur le terrien, $T$ :

\begin{tabular}{|c|c|c|}
\hline & Tn'entend pas & T entend \\
\hline $\begin{array}{c}\mathrm{E} 0, \mathrm{~N}=62 \\
\text { troisième }\end{array}$ & $\mathbf{6 5} \%$ & $6 \%$ \\
\hline
\end{tabular}

\section{Encadré $\mathrm{n}^{\circ} 9$}


Enoncé: (version 1) : 7 tubes identiques, 7 haut-parleurs et 7 micros identiques :

HP

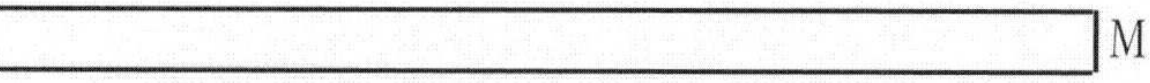

air ( $P$ et $T$ différents), hydrogène, eau, acier, tube où on a fait le vide.

43 Tous les haut-parleurs commencent à émettre un « la » au même instant et s'arrêtent au même instant.

question1: Est-ce que les micros enregistrent un son? Oui, lesquels, pourquoi? Non, pourquoi?

question 2: Pour les micros qui enregistrent un son, commencent-ils à enregistrer au même instant? Oui, pourquoi? Non, pourquoi? Classer les micros par ordre d'arrivée croissant du son.

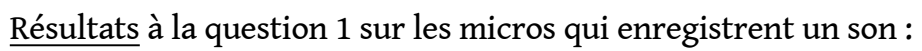

\begin{tabular}{|c|c|c|c|c|}
\hline $\begin{array}{l}\mathrm{E} 0, \mathrm{~N}=39, \\
\text { seconde (version } 1) \\
E 0, N=26 \text {, } \\
\text { troisième (version 2) }\end{array}$ & \multicolumn{2}{|c|}{ enregistre } & \multicolumn{2}{|c|}{ n'enregistre pas } \\
\hline vide & $74 \%$ & $31 \%$ & $13 \%$ & $54 \%$ \\
\hline gaz & $88 \%$ & $69 \%$ & $3 \%$ & $15 \%$ \\
\hline eau & $54 \%$ & $77 \%$ & $36 \%$ & $15 \%$ \\
\hline acier & $36 \%$ & $23 \%$ & $49 \%$ & $62 \%$ \\
\hline
\end{tabular}

Résultats à la question 2 sur le classement des vitesses de propagation :

\begin{tabular}{|l|c|c|}
\hline $\mathrm{E} 0, \mathrm{~N}=15$, seconde & "lent" & "rapide" \\
\hline vide & $\mathbf{3 3} \%$ & $47 \%$ \\
eau & $60 \%$ & $\mathbf{7} \%$ \\
acier & $53 \%$ & $\mathbf{0} \%$ \\
\hline
\end{tabular}




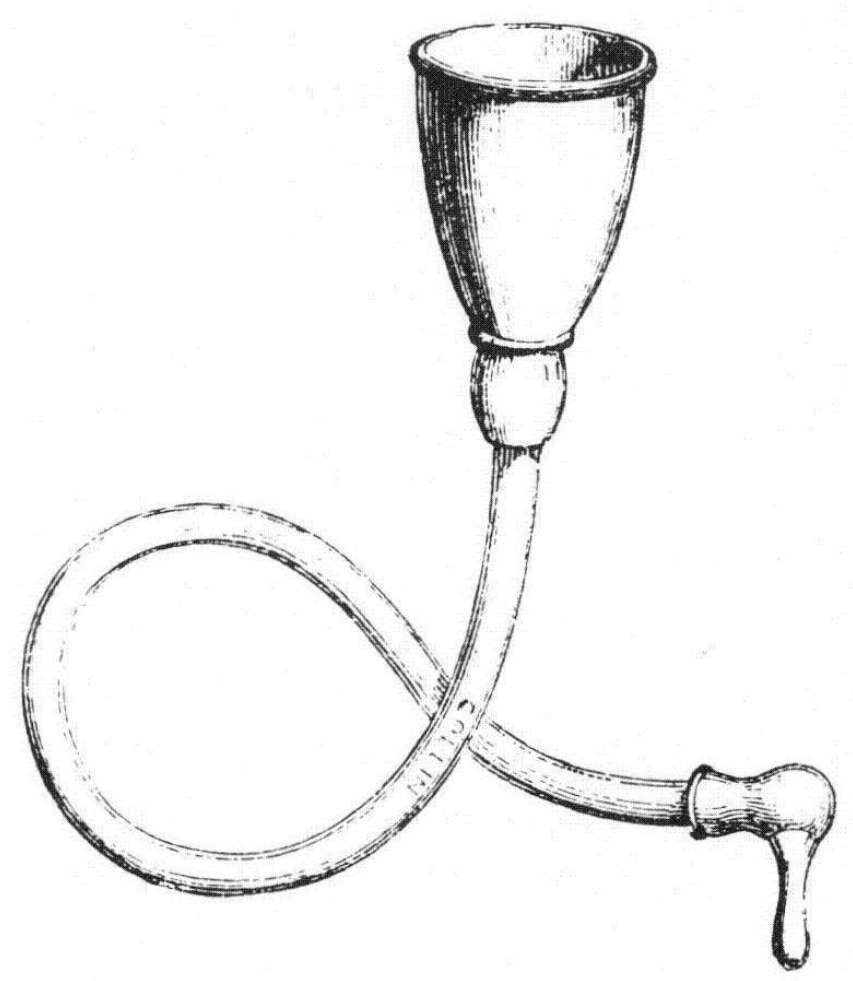

Cornet acoustique

\section{BIBLIOGRAPHIE}

\section{Références}

MAUR1NES L. : « Premières notions sur la propagation des signaux mécaniques : analyse des difficultés des étudiants » Nouvelle thèse. Paris VII, 1986

MAURINES L : « Spontaneous reasoning on the propagation of visible mechanical signals » Int.J.Sci.Educ, vol 14, n 14, pp 279-293, 1992.

VIENNOT L. : « Spontaneous reasoning in elementary dynamics » Eur.J.Sci.Educ, vol 1, n², pp 205-221, 1979.

SALTIEL E. et MALGRANGE J-L. : « Spontaneous ways of reasoning in elementary Kinematics », Eur.J.of Phys.,vol 1, p 73, 1980.

CLOSSET J-L. : « Les obstacles à l'apprentissage de l'électrocinétique », B.U.P., n7l6, Paris, pp 931-949, 1989.

ROZIER S. et VIENNOT L. : « Students' reasoning in thermodynamics » Int.J.Sci.Educ, vol 13, n², pp 159-170, 1991. 


\section{NOTES}

1. Termes soulignés par l'auteur (NDLR)

\section{RÉSUMÉS}

Cette communication présente les résultats d'une enquête exploratoire visant à analyser les conceptions d'élèves sur la propagation d'un signal sonore. On rencontre les mêmes tendances vers un raisonnement mécaniste et mononotionnel que celles mises en évidence pour la propagation d'un signal sur une corde. La source crée et met en mouvement un « signal - objet», matérialisation d'un capital qu'elle lui communique. Ce capital, mélange de force, d'énergie, de vitesse est l'unique support du raisonnement spontané. 1 conduit à une interdépendance entre ce qui se passe en un point du milieu et la vitesse de propagation: celle-ci dépend de la source, de l'amplitude du signal et peut diminuer au cours du temps. Le milieu est un support passif voire inutile : le son peut se propager dans le vide.

The results of a preliminary enquiry regarding 16 years' old children's conceptions on propagation of sound are presented. The same tendencies towards a mechanistic and mononotional reasoning appear as those put forward for the propagation of a signal on a string. The signal is a material object created and set in motion by the source. It materializes a "supply» given to it by the source. This supply, a mixture of force, energy, speed, is the base of the spontaneous reasoning. It leads to a link between the propagation velocity and what happens at a given point of the medium: this velocity depends on the source, on the signal amplitude and can decrease with time. The medium is a passive support even useless : sound can propagate in the vacuum.

\section{INDEX}

Mots-clés : onde, propagation, raisonnement spontané, signal, son

\section{AUTEUR}

\section{MAURINES}

LDPES, Université de Paris VII 